\title{
OPEN Examining potential confounding factors in gene expression analysis of human saliva and identifying potential housekeeping genes
}

\author{
P. Ostheim ${ }^{1 凶}$, S. W. Alemu ${ }^{1}$, A. Tichý' ${ }^{2,3}$, I. Sirak ${ }^{4}$, M. Davidkova ${ }^{5}$, M. Markova Stastna ${ }^{6}$, \\ G. Kultova ${ }^{2}$, S. Schuele ${ }^{1}$, T. Paunesku' , G. Woloschak' ${ }^{7}$, S. A. Ghandhi ${ }^{8}$, S. A. Amundson ${ }^{8}$, \\ M. Haimerl ${ }^{9}$, C. Stroszczynski ${ }^{9}$, M. Port ${ }^{1} \&$ M. Abend ${ }^{1}$
}

Isolation of RNA from whole saliva, a non-invasive and easily accessible biofluid that is an attractive alternative to blood for high-throughput biodosimetry of radiological/nuclear victims might be of clinical significance for prediction and diagnosis of disease. In a previous analysis of 12 human samples we identified two challenges to measuring gene expression from total RNA: (1) the fraction of human RNA in whole saliva was low and (2) the bacterial contamination was overwhelming. To overcome these challenges, we performed selective CDNA synthesis for human RNA species only by employing poly(A)+-tail primers followed by qRT-PCR. In the current study, this approach was independently validated on 91 samples from 61 healthy donors. Additionally, we used the ratio of human to bacterial RNA to adjust the input RNA to include equal amounts of human RNA across all samples before CDNA synthesis, which then ensured comparable analysis using the same base human input material. Furthermore, we examined relative levels of ten known housekeeping genes, and assessed inter- and intra-individual differences in 61 salivary RNA isolates, while considering effects of demographical factors (e.g. sex, age), epidemiological factors comprising social habits (e.g. alcohol, cigarette consumption), oral hygiene (e.g. flossing, mouthwash), previous radiological diagnostic procedures (e.g. number of CT-scans) and saliva collection time (circadian periodic). Total human RNA amounts appeared significantly associated with age only $(P \leq 0.02)$. None of the chosen housekeeping genes showed significant circadian periodicity and either did not associate or were weakly associated with the 24 confounders examined, with one exception, $60 \%$ of genes were altered by mouthwash. ATP6, $A C T B$ and $B 2 M$ represented genes with the highest mean baseline expression (Ct-values $\leq 30)$ and were detected in all samples. Combining these housekeeping genes for normalization purposes did not decrease inter-individual variance, but increased the robustness. In summary, our work addresses critical confounders and provides important information for the successful examination of gene expression in human whole saliva.

Over the last two decades, saliva has become of increased interest as an easily accessible and non-invasive source of human biomarkers. Besides DNA, proteins and various metabolites, RNA (mRNA and miRNA species) has also been shown to be a promising marker in all tissues and body fluids ${ }^{1-4}$. Because saliva is derived from several tissue sources and also contains large amounts of total RNA it is one of the most attractive diagnostic, prognostic, and monitoring tools for both systemic and oral diseases ${ }^{5-7}$. As such, saliva has been shown to contain RNA

\footnotetext{
${ }^{1}$ Bundeswehr Institute of Radiobiology affiliated to the University of Ulm, Neuherbergstr. 11, 80937 Munich, Germany. ${ }^{2}$ Department of Radiobiology, Faculty of Military Health Sciences in Hradec Kralove, University of Defence in Brno, Brno, Czech Republic. ${ }^{3}$ Biomedical Research Centre, University Hospital, Hradec Králové, Czech Republic. ${ }^{4}$ Department of Oncology and Radiotherapy, University Hospital and Medical Faculty in Hradec Kralove, Hradec Králové, Czech Republic. ${ }^{5}$ Department of Radiation Dosimetry, Nuclear Physics Institute of the Czech Academy of Sciences, Prague, Czech Republic. 'Institute for Hematology and Blood Transfusion, Hospital Na Bulovce, Prague, Czech Republic. ${ }^{7}$ Department of Radiation Oncology, Northwestern University, Chicago, IL 60611, USA. ${ }^{8}$ Center for Radiological Research, Columbia University Irving Medical Center, New York, NY 10032, USA. ${ }^{9}$ Department of Radiology, University Hospital Regensburg, Regensburg, Germany. ${ }^{\boxplus}$ email: patrickostheim@bundeswehr.org
} 
biomarkers for prediction and diagnosis of several diseases especially of the oral cavity such as oral cancer ${ }^{8-10}$ and disorders of the salivary glands ${ }^{11,12}$. Saliva represents information from several bodily sources, including blood, because saliva is a plasma ultra-filtrate. This means that most compounds found in blood are also in saliva, leading to the aphorism that saliva is a "mirror of the body"13,14.

Also, collection of saliva samples represents an easy, cheap, non-invasive alternative to blood collection. In a previous study, we identified two problematic issues not coherently described before when working with human whole saliva: (1) the fraction of human RNA in whole saliva was low and (2) the bacterial contamination was overwhelming ${ }^{15}$. The previous study was based on 12 samples and served as an initial "proof-of-concept" study. Following this, in the current work, we have modified the methodology to (1) select only human RNA during cDNA synthesis by targeting the poly(A)+-tailed mRNA and (2) introduced pre-amplification of human RNA before qRT-PCR ${ }^{15}$. The combination of these two important modifications to our protocols resulted in sufficient amounts of high quality and quantity human RNA. In this context, we also discussed numerous advantages of collecting whole saliva as a source for RNA biomarkers instead of focusing on salivary supernatant ${ }^{15}$.

Not being a sterile medium like blood, saliva can be affected by many confounding variables, which then influence the quality and quantity of RNA that can be isolated from human whole saliva. The aim of this manuscript is to examine if there are any other prerequisites that have to be considered for satisfying results in this approach e.g. the optimal saliva sampling time or the impact of potential confounders such as demographics (e.g. sex, age) or epidemiological factors comprising social habits (e.g. alcohol, cigarette consumption), oral hygiene (e.g. flossing, mouthwash) or previous radiological diagnostic procedures (e.g. number of computed tomography/CTscans). In the current exploratory study, we addressed the following aspects/tasks (Fig. 1): (I) For methodological reasons, we applied the previously described workflow ${ }^{15}$ using 91 instead of 12 samples (for validation purposes) and introduced the adaption of human RNA input for cDNA-synthesis to ensure equal human RNA amounts and improved comparability among different samples. (II) We examined inter- and intra-individual differences in salivary isolates considering potential demographic and epidemiological confounding factors $(\mathrm{n}=24)$ as well as different saliva collection time points. (III) Human $18 \mathrm{~S}$ rRNA, as a commonly known housekeeping gene, cannot be used for normalization purposes in gene expression analysis in the current application due to its lack of a poly-A-tail ${ }^{15}$. We examined baseline gene expression values of ten commonly used housekeeping genes in human whole saliva to accomplish the above mentioned tasks and present our analysis here.

\section{Materials and methods}

Sample collection. Whole saliva samples were collected using ORAgene RNA (RE-100, catalog number: RE-100) vial collection kits from DNA Genotek according to the manufacturer's instructions (DNA Genotek Inc., Kanata, Ontario, Canada). The kit is an all-in-one system for the collection, stabilization and transportation of RNA from saliva. Unstimulated whole saliva was collected from 61 healthy donors ( 27 females, 34 males, average age $38.5 \pm 16.4$ years, Fig. 1). The following exclusion criteria were applied: age below 18 years old, donors with history of immunodeficiency, autoimmune disorders, viral hepatitis, HIV infection, current or previous cancer, current oral problems (infection). From all donors, whole saliva was collected from 9 to 11 am and then preserved after having been shaken vigorously in the vial. Additionally, in 10 out of 61 donors, whole saliva samples were collected at $9 \mathrm{am}, 3 \mathrm{pm}, 9 \mathrm{pm}$ and 9 am again the next day. After completing normal oral hygiene, donors were not allowed to eat or smoke $2 \mathrm{~h}$ prior to collection or to drink at least $1 \mathrm{~h}$ prior to collection. Samples were stored at room temperature overnight and placed in a freezer $\left(-20^{\circ} \mathrm{C}\right)$ for storage. All samples were anonymized and obtained with informed consent from the donors. Sampling was carried out in accordance with the institutional guidelines and regulations. At the time of sampling, donors were asked to fill in a questionnaire (supplemental Figure 1) about demographics (e.g. sex, age), social habits (e.g. alcohol, smoking), oral hygiene (e.g. flossing, mouth wash), and previous radiological diagnostic procedures (e.g. number of CT-scans).

RNA extraction. Total RNA comprising a mixture of human and bacterial RNA, was isolated from whole saliva samples following a combination of the ORAgene ${ }^{\oplus}$ RNA purification protocol ${ }^{16}$ and the mirVana ${ }^{\mathrm{Tw}}$ kit protocol (Invitrogen ${ }^{\mathrm{Tu}}$, ThermoFisher Scientific, Carlsbad, CA 92008; USA/Life Technologies, Darmstadt, Germany) as described in detail elsewhere ${ }^{15}$. In brief, the samples were heated at $50^{\circ} \mathrm{C}(1 \mathrm{~h}$ ), three aliquots (of $1000 \mu \mathrm{l})$ were generated, incubated at $90^{\circ} \mathrm{C}(15 \mathrm{~min})$, cooled to room temperature, $40 \mu$ l ORAgene ${ }^{\oplus}$ neutralizer solution $(1 / 25$ of total volume) was added, incubated on ice, centrifuged at $13,000 \mathrm{~g}(3 \mathrm{~min})$ and the cell-free clear supernatant was collected for further processing. At this step, we switched to the mirVana ${ }^{\mathrm{m}}{ }^{\mathrm{m}}$ kit protoco ${ }^{17}$ by adding the Lysis/ Binding Solution. With the mirVana ${ }^{\mathrm{Tm}}$ kit, total RNA, including human and bacterial RNA species, was isolated by combining a Phenol-Chloroform RNA precipitation with further processing using silica membranes. After several washing procedures to purify RNA from other residual debris, DNA residuals were digested on the membrane (RNAse-free DNAse Set, Qiagen, Hilden, Germany). RNA was eluted with $100 \mu$ l RNAse free water in a collection tube and the aliquots were pooled for each sample. In order to increase the input RNA amount for downstream gene expression analysis, samples were steamed at $45^{\circ} \mathrm{C}$ for 90 min followed by elution with $30 \mu \mathrm{l}$ of RNase free water before freezing at $-20^{\circ} \mathrm{C}$.

Quality and quantity of isolated total RNA were measured spectrophotometrically using NanoDrop ${ }^{\mathrm{rw}}$ One Microvolume UV-Vis spectrophotometer (NanoDrop, PeqLab Biotechnology, Erlangen, Germany). RNA integrity was assessed by the 2100 Agilent Bioanalyzer (Life Science Group, Penzberg, Germany) and DNA contamination was controlled by conventional PCR using actin primers.

Conventional cDNA synthesis-high-capacity cDNA reverse transcription kit. For analyzing gene expression of human rRNA (18S) and pan-bacterial rRNA (16S, see below), total salivary RNA was converted into complementary DNA (cDNA) via reverse transcription using the High-capacity cDNA reverse tran- 


\section{Experiment}

- $n=61$ healthy donors

- 34 males $(\mathrm{m})$

- 27 females (f)

- Saliva samples:

\begin{tabular}{|c|c|c|c|c|c|}
\hline \multirow[b]{2}{*}{ donors } & \multirow[b]{2}{*}{$\begin{array}{l}\text { time points of } \\
\text { sampling }\end{array}$} & \multicolumn{4}{|c|}{ Number of samples for } \\
\hline & & Task I & $\begin{array}{l}\text { Tas } \\
\mathbf{a} / \mathbf{b}\end{array}$ & & Task II \\
\hline 51 donors & $9 \mathrm{am}$ & 51 & 51 & & \\
\hline \multirow{4}{*}{10 donors } & $9 \mathrm{am}$ & 10 & 10 & 10 & 10 \\
\hline & $3 \mathrm{pm}$ & 10 & & 10 & 10 \\
\hline & $9 \mathrm{pm}$ & 10 & & 10 & 10 \\
\hline & 9 am next day & 10 & & 10 & 10 \\
\hline & & 91 & 61 & 40 & 40 \\
\hline
\end{tabular}

- 51 donors á one saliva sample (taken 9am), $\mathrm{n}=51$ samples

- 10 donors á four saliva samples (9am, 3pm, 9pm and 9am next day), $\mathrm{n}=40$ samples

$\rightarrow 61$ donors, 91 samples in total

\section{Task I - Methodological considerations ( $\mathrm{n}=61$ donors, $\mathrm{n}=91$ samples)}

1. Modifying the established workflow for performing robust gene expression analysis in salivary samples

2. Overcoming two problematic issues:

$\rightarrow$ overwhelming bacterial contamination

$\rightarrow$ human RNA of low abundance

3. Main modification: $18 \mathrm{~S} / 16 \mathrm{~S}$ rRNA ratio calculation and adj ustment of human RNA input for cDNA synthesis

\section{Task II - Identifying inter- and intra-individual differences in saliva isolates ( $n=61 / 10$ donors, $n=61 / 40$ samples)}

Ila/b: Influence of sociodemographic characteristics (sex, age) and epidemiological characteristics (social habits, oral hygiene and previous radiological procedures, see table 1) on saliva RNA endpoints ( $n=61$ donors, $n=61$ samples)

Ilc: Correlation of human/bacterial RNA with a circadian periodic of saliva collection

4 samples in 10 donors ( $n=10$ donors, $n=40$ samples):

- $\quad 9 a m(0 h)$

- $\quad 3 p m(6 h)$

- $\quad 9 p m(12 h)$

- $\quad 9 a m$ next day $(24 \mathrm{~h})$

Endpoints: RNA quality \& quantity, bacterial contamination (18S/16S rRNA), amount of human RNA (18S rRNA)

\section{Task III - Housekeeping genes ( $\mathrm{n}=10$ donors, $\mathrm{n}=40$ samples)}

1. Baseline gene expression values of potential housekeeping genes for normalization purposes at four time points:

- $\mathrm{n}=10$ donors (8 males, 2 females)

- each donor with 4 sampling time points (9am, 3pm, 9pm and 9am next day): $n=40$ samples

2. Correlation of housekeeping gene expression with sociodemographic and epidemiologic characteristics

3. Correlation of housekeeping gene expression with a circadian periodic of saliva collection

Figure 1. Overview of the samples, study design and tasks I-III description. The inserted table shows the number of samples used in the different tasks as well as the time points of sampling.

scription kit ${ }^{18}$ (Applied Biosystems ${ }^{\mathrm{m}}$, Life Technologies, Darmstadt, Germany). The amount of total RNA input was always determined to $500 \mathrm{ng}$ measured by NanoDrop ${ }^{\mathrm{Tn}}$ One. After reverse transcription, cDNA was diluted to a concentration of $0.01 \mathrm{ng} / 10 \mu \mathrm{l}$, which was used for qRT-PCR detection of $16 \mathrm{~S}$ and $18 \mathrm{~S} \mathrm{rRNA}$.

Adjustment of human RNA input for cDNA synthesis. A main modification of the previously described workflow ${ }^{15}$ was the adaption of human RNA input for cDNA synthesis with the SuperScript ${ }^{\circledast}$ III FirstStrand Synthesis System (Fig. 2). As NanoDrop ${ }^{\text {Tw }}$ spectrophotometer only provides total RNA values comprising an unknown mixture of human and bacterial RNA species, we calculated the ratio of the detected raw Ct-values (threshold cycles) of human rRNA (18S) to pan-bacterial rRNA (16S, high-capacity cDNA reverse transcription kit cDNA synthesis followed by 1st qRT-PCR), regarding rRNA as representative for all human and bacterial RNA specimens. The ratio was determined by calculating the relative ratio of $18 \mathrm{~S}$ rRNA to $16 \mathrm{~S}$ rRNA for each sample individually as follows:

Fold change $=2^{\wedge(\mathrm{Ct} 18 \mathrm{~S} \text { rRNA }-\mathrm{Ct} 16 \mathrm{~S} \text { rRNA })}$ 


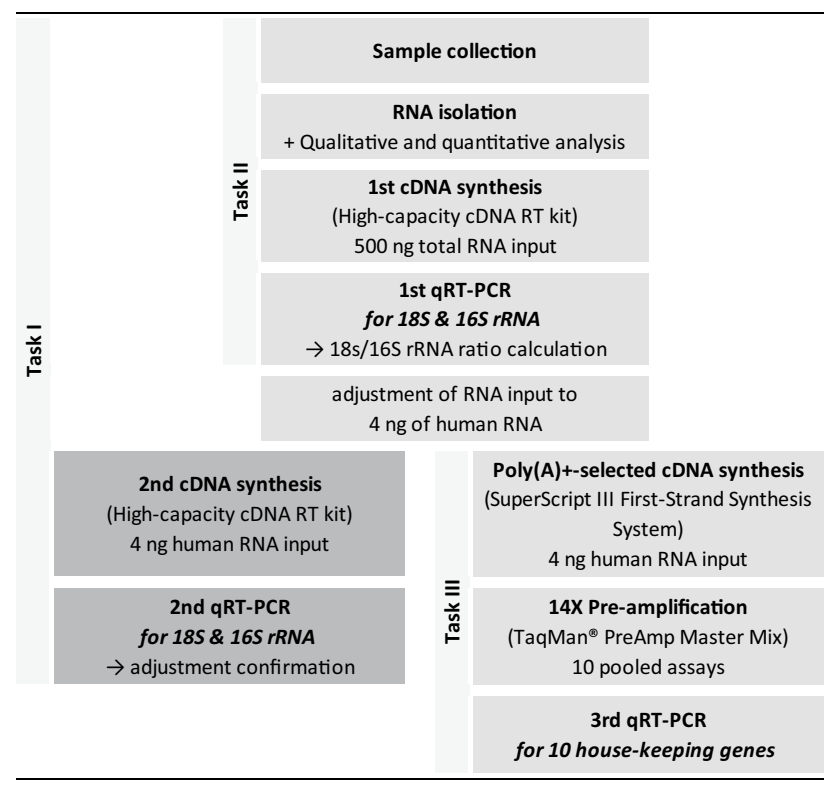

Figure 2. The flow chart displays the different steps (rows) in gene expression analysis including our modified workflow, the tasks, the required kits and the detour for adjustment of human RNA input as well as its confirmation (boxes in darker grey). The boxes in brighter grey depict the advanced methodological workflow for gene expression analysis in whole saliva samples.

Using the generated ratio, a RNA input of 4 ng total human RNA was determined individually for each sample for a second cDNA synthesis followed by 2nd qRT-PCR (confirmation for methodological reasons in this context, Fig. 2 boxes in darker grey).

Modified cDNA synthesis-SuperScript III First-Strand Synthesis System. In order to subsequently perform gene expression analysis of human origin, only eukaryotic messenger RNA (mRNA) was reverse transcribed to cDNA by using the SuperScript ${ }^{\circ}$ III First-Strand Synthesis System with Oligo (dT) ${ }_{20}$ prim- $^{-}$ ers. As a result, pan-bacterial RNA and other human RNA species were excluded from further processing, minimizing the inhibition effects of those RNA species on the following reactions. According to the kit description, the amount of starting material can vary from $1 \mathrm{pg}$ to $5 \mu \mathrm{g}$ of RNA and the maximum input volume of RNA is $8 \mu \mathrm{l}^{19}$. The amount of human RNA input was set to $4 \mathrm{ng}$ per reaction. Using the concentration from repeated NanoDrop $^{\text {tw }}$ measurements and the calculated $18 \mathrm{~S} / 16 \mathrm{~S}$ ratio in each sample, the corresponding amount of measured total RNA input was calculated, conforming to the maximum input volume of $8 \mu$ l. The RT was performed according to the manufacturer's instructions ${ }^{19}$.

Pre-amplification-TaqMan ${ }^{\circledR}$ PreAmp Master Mix. To detect low abundance mRNA species, preamplification was required. We used TaqMan PreAmp Master Mix (Thermo Fisher Scientific Baltics UAB, Vilnius, Lithuania) to increase the amount of specific cDNA targets, synthesized with the SuperScript ${ }^{\oplus}$ III First-Strand Synthesis System. According to the manufacturer, pre-amplification with this kit is linear when a minimum amount of cDNA molecules is present (minimum of 1-250 ng and Ct-values without pre-amplification should be $<35$ ) and multiplex amplification can be performed by pooling up to 100 TaqMan ${ }^{\otimes}$ Gene Expression Assays. Pre-amplification was performed according to the TaqMan ${ }^{\circledR}$ PreAmp master mix kit protocol ${ }^{20}$. In the present work, 10 different TaqMan ${ }^{\bullet}$ Gene Expression Assays (ACTB, Hs01060665_g1; B2M, Hs00187842_m1; GUSB, Hs00939627_m1; MT-ATP6, Hs02596862_g1; PGK1, Hs00943178_g1; PP1A, Hs99999904_m1; RPL13A, Hs04194366_g1; RPLP0, Hs02992885_s1; TBP, Hs00427620_m1; YWHAZ, Hs01122445_g1) were utilized and pooled to enable the multiplex amplification of specific cDNA targets. Those are commonly used house-keeping genes already employed in other experimental set ups (www.genomics-online.com).

Real-time quantitative reverse transcription polymerase chain reaction (qRT-PCR). Using different sets of primers, two kinds of cDNAs were utilized for qRT-PCR: for human (18S rRNA, Hs99999901_g1) and pan-bacterial (16S rRNA, Ba04230899_s1) primer probe designs, cDNA from High-capacity cDNA reverse transcription kit was used, whereas for the primer probe designs representing the potential house-keeping genes

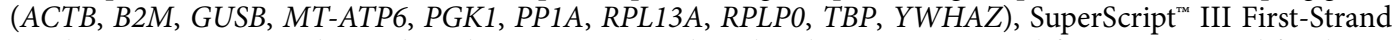
Synthesis SuperMix synthesized, i.e. human cDNA with and without $14 \times$ pre-amplification was used for detection of each of these genes in each sample. The qRT-PCR reaction contained TaqMan ${ }^{\star}$ Universal PCR Master Mix and one of the inventoried TaqMan ${ }^{\oplus}$ Gene Expression Assays for separate detection of transcripts. All meas- 
urements were run in duplicate, using a 96-well-format TaqMan ${ }^{\circ}$ qRT-PCR platform and the QuantStudio ${ }^{\mathrm{mn}} 12 \mathrm{~K}$ Flex Real-Time PCR System.

Statistical analysis. Descriptive statistics (n, mean, standard deviation, minimum [min], maximum [max]) were calculated for continuous variables such as gene expression data and age. Frequency tables of categorical data were examined for statistical differences using the chi-square test for equal proportion. Comparisons of categorical variables with gene expression values were performed using the non-parametric Kruskal-Wallis (KW) test. We assessed the assumptions of normality (Kolmogorov-Smirnov) and boxcox transformed the continuous variable "age". All calculations were performed using SAS (release 9.4, Cary NC, USA). Graphical presentations were performed using Sigma Plot 14 (Jandel Scientific, Erkrath, Germany).

Data availability and approval statement. The merged set of raw data is provided within supplemental Table 2. The qRT-PCR related measurements (e.g. RNA quantity/quality and TaqMan ${ }^{\circledR}$ qRT-PCR) were performed according to the standard operating procedures implemented in our laboratory in 2008 when the Bundeswehr Institute of Radiobiology became DIN-certified by TÜV Süd München, Germany (DIN EN ISO 9001/2008). All samples and data was processed anonymously without exception and only for this specific purpose. All data is handled according the European General Data Protection Regulation. Data will be deleted after 10 years. Due to the minimal-invasive collection and the fully anonymized processing of the samples, the local ethical commission (Ethics committee, Bayerische Landesärztekammer, Munich, Germany) decided that experiments can be performed in agreement with ethical standards and do not require an additional approval.

\section{Results}

Methodological considerations. RNA isolation. From 91 samples an average of $93.3 \mu \mathrm{g}(\mathrm{SD} \pm 141.7)$ total RNA per $2 \mathrm{ml}$ saliva could be isolated. Moreover, high purity RNA with $\mathrm{OD}_{260 / 280}$ ratios at a mean of 2.1 was isolated from saliva. A mean RNA integrity number (RIN) of $5.9(\mathrm{SD} \pm 1.4)$ was detected for saliva samples and all saliva samples showed gel-like image bands of human $28 \mathrm{~S}$ and $18 \mathrm{~S}$ rRNA that did not provide any indications for severe degradation. No DNA contamination could be detected by beta actin PCR in all samples (data not shown).

Ratio of human to bacterial RNA. Human $18 \mathrm{~S}$ rRNA and pan-bacterial $16 \mathrm{~S}$ rRNA raw Ct-values were measured from whole saliva via qRT-PCR following a first cDNA-synthesis with the High-capacity Kit (total RNA input of $500 \mathrm{ng}$ ). A mean human $18 \mathrm{~S}$ rRNA raw Ct-value of 28.5 (SD \pm 4.9 , min 20.4, $\max 35.7$ ) and a mean bacterial $16 \mathrm{~S}$ rRNA raw Ct-value of 17.1 (SD \pm 1.04 , min 15.9, max 19.7) implied on average about 2,702 (2 $\left.2^{28.5-17.1}\right)$ times more bacterial RNA copy numbers relative to human RNA in whole saliva (Fig. 3A). This means that for each copy of a human gene, on average 2,702-times more copies of bacterial genes can be found in the samples. Human $18 \mathrm{~S}$ rRNA raw $\mathrm{Ct}$ values showed a broad variance with almost $10 \mathrm{Ct}$ values in the $50 \%$ interquartile range, indicating about 1000 -fold differences in RNA copy numbers (Fig. 3A).

Adjustment of human RNA input for cDNA-synthesis and its confirmation. Using the generated ratio

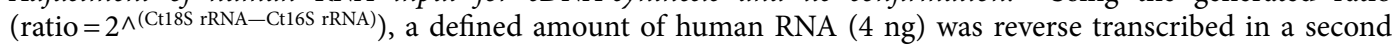
cDNA synthesis, again using High-capacity cDNA reverse transcription kit, followed by qRT-PCR analysis for detection of human $18 \mathrm{~S}$ rRNA and bacterial $16 \mathrm{~S}$ rRNA Ct-values (Fig. 3B). The $50 \%$ interquartile range of human 18S rRNA quantification in 51 saliva samples changed significantly $(P<0.001)$ from 7 Ct values of unadjusted input RNA (comprising an unknown mixture of human and bacterial RNA) to $1.5 \mathrm{Ct}$ values after considering the degree of bacterial contamination and adjusting for it.

Identifying inter- and intra-individual differences in saliva RNA isolates. Sociodemographic and epidemiological characteristic of donors. The 61 healthy donors comprised almost equal proportions of females (44.3\%) and males (55.7\%, Table 1). Donors were mostly Caucasians (77.1\%) and about half of them were aged 19-30 years at time of saliva collection. Only $18 \%$ smoked over at least 5 years and another $16 \%$ of former smoker smoked at least 2 years and stopped smoking about a year ago. Alcohol consumption and diet was reported by $41 \%$ and $28 \%$, respectively. Most (83.6\%) of the donors brushed their teeth at least twice a day and about half of them used flossing and about one-third mouthwash. Braces (3.3\%) and dentures (11.5\%) were reported less frequently and oral problems like periodontitis in $23 \%$. Acute and chronic diseases such as rheumatism or disease of the thyroid gland were reported in $6.6 \%$ and $16.4 \%$, respectively. Radiological examinations during the last six months (mainly X-rays and CT-scans) were reported in $18 \%$ and none of our donors received radiotherapy.

Correlation of human/bacterial RNA with sociodemographic and epidemiological characteristics. Total RNA concentration increased almost three-fold with higher alcohol consumption (data not shown). Only human RNA (18S rRNA) amount as well as the ratio of $18 \mathrm{~S} / 16 \mathrm{~S}$ rRNA appeared significantly associated with age on a categorical $(P=0.07,0.04)$ or linear scale $(P=0.02,0.02)$, which was not shown for bacterial RNA (16S rRNA). We built two groups based on relatively high $(18 \mathrm{~S}$ rRNA Ct value $\leq 30)$ and relatively low $(18 \mathrm{~S}$ RNA Ct value $>30)$ amounts of human RNA. Within the younger age group $(<30$ years) the mean human RNA amount (18S rRNA as representative) was 676-fold higher between both human 18S rRNA groups, but neither sociodemographic nor epidemiological parameters appeared significantly associated (data not shown). In the older ( $>30$ years old) compared to younger donor group ( $\leq 30$ years old), examinations regarding the high yield human $18 \mathrm{~S}$ rRNA 

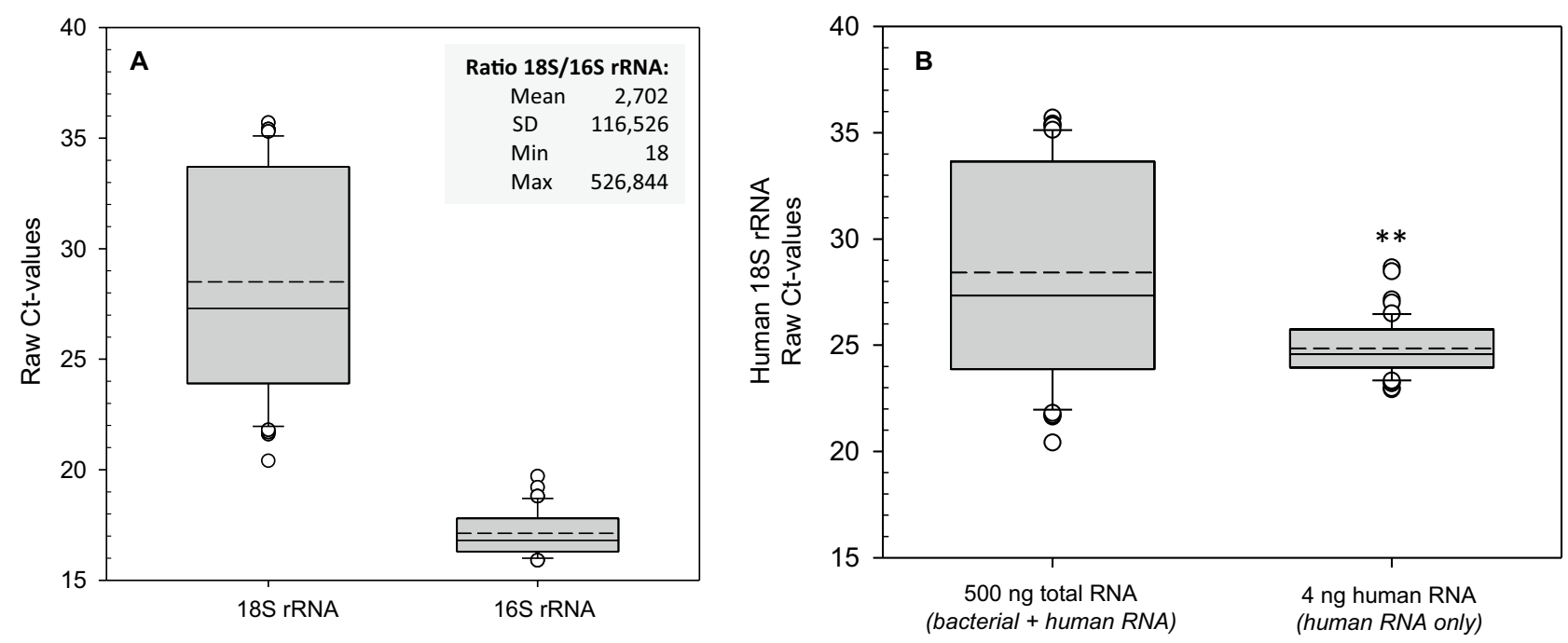

Figure 3. The box plots in (A) display the human $18 \mathrm{~S}$ rRNA and bacterial $16 \mathrm{~S}$ rRNA raw Ct values (threshold cycles) for all whole saliva samples $(n=91)$. Dashed lines represent the mean, solid lines the median and dots the outliers. The input amount for cDNA synthesis for each sample was $0.5 \mu \mathrm{g}$. The inserted table shows the calculated ratio between raw $\mathrm{Ct}$ values of human $18 \mathrm{~S}$ rRNA and bacterial $16 \mathrm{~S}$ rRNA and provides descriptive statistics: mean, minimum [min], maximum [max], standard deviation [stdev] and standard error of the mean [sem]. The box plots in (B) represent the human 18S rRNA raw Ct-values before and after adjustments accounting for input differences from left to right. The left part shows Ct values from 1st qRT-PCR performed using cDNA with an input of $500 \mathrm{ng}$ total RNA (bacterial and human RNA) and the right boxplot the corresponding results when taking $4 \mathrm{ng}$ of human RNA (calculated via the $18 \mathrm{~S} / 16 \mathrm{~S}$-ratio together with total RNA concentration values measured). Asterisks ${ }^{(* *}$ ) refer to a $P$ value $<0.001$ using $500 \mathrm{ng}$ total RNA measurements as reference.

group showed significantly more alcohol consumption per week $(P=0.012)$, higher frequency of former smoker $(P=0.0058)$, radiological examinations $(P=0.012)$ and dentures $(P=0.032)$.

Correlation of human/bacterial RNA with a circadian periodic saliva collection. Among all time points, the amount of bacterial RNA was constantly high (mean raw Ct values of bacterial 16S rRNA were 17.0-17.7) with a small variance (SD raw Ct values of bacterial 16S rRNA 0.9-1.1 over all time points) whereas the amount of human RNA was lower (mean raw Ct values of human 18S rRNA 28.0-29.6) with a large variance (SD raw Ct values of human 18S rRNA 4.0-5.2 over all time points, Fig. 4A). The intra-individual variance of human $18 \mathrm{~S}$ rRNA was very high in most of the samples with a SD of up to 5.8 among the time points. In donor 1, we observed a minimum human 18S rRNA raw Ct value of 21.7 (9 am the next day) and a maximum 18S rRNA raw $\mathrm{Ct}$ value of $35.1(3 \mathrm{pm})$, resulting in a delta $\mathrm{Ct}$ value of more than 13 or in other words more than 8,000 fold difference in human RNA amount (data not shown). Relative to the earliest sampling time at 9 am, slightly increased median RIN values and about two-fold decreased interquartile ranges were observed at 3 and $9 \mathrm{pm}$ (Fig. 4B, $P=0.022$ ), but distribution of RIN values at 9 am of the following day were similar to the 9 am values of the previous day (Fig. 4B).

Identifying inter- and intra-individual differences in housekeeping genes. Baseline gene expression values of housekeeping genes. Among ten examined housekeeping genes, seven (GUSB, PGK1, PP1A, RP$L 13 A, R P L P 0, T B P, Y W H A Z$ ) revealed baseline levels $>30$ Ct-values without pre-amplification (Fig. 5). Three genes revealed Ct-values $<30$ without pre-amplification, namely ATP6 (mean raw Ct value $=25.9)$, ACTB (mean raw $C t$ value $=28.6)$ and $B 2 M$ (mean raw $C t$ value $=29.8$ ) indicating high expression levels and detection in all samples (Figs. 5, 6, supplemental Table 1, supplemental Table 2).

Correlation of housekeeping gene expression with sociodemographic and epidemiological characteristics. Eight of ten housekeeping genes showed a weak $(P=0.02-0.05)$ but significant association of altered gene expression and oral hygiene like mouth wash and flossing (Table 2). Further weak associations were found for alcohol consumption with PGK1 $(P=0.03)$, age with RPLPO $(P=0.034)$ and radiological examination during the last 6 months with ATP6 gene expression $(P=0.046)$. Only $T B P$ showed no significant association with sociodemographic and epidemiological characteristics (Table 2).

Correlation of housekeeping gene expression with a circadian periodic of saliva collection. None of the housekeeping genes revealed significant gene expression changes associated with the time of saliva sampling, but different patterns in gene expression changes over time of sampling were observed for individuals (Fig. 6). For example, we found a decrease of normalized Ct values among all genes in donor 9 at 9 am on the next day, 


\begin{tabular}{|c|c|c|c|c|}
\hline Parameter & categories & Number $(n=61)$ & percent & Pvalue \\
\hline \multicolumn{5}{|l|}{ Demographic characteristics } \\
\hline \multirow{2}{*}{ Sex } & Female & 27 & 44.3 & \\
\hline & Male & 34 & 55.7 & 0.4 \\
\hline \multirow{2}{*}{ Race } & Caucasian & 47 & 77.1 & \\
\hline & Other & 14 & 23.0 & $<0.0001$ \\
\hline \multirow{9}{*}{ Age (years) } & $\geq 19-30$ & 30 & 49.2 & \\
\hline & $>30-50$ & 15 & 24.6 & \\
\hline & $>50$ & 16 & 26.2 & $<0.0001$ \\
\hline & $\mathrm{n}$ & 61 & & \\
\hline & Mean & 38.5 & & \\
\hline & Stdev & 16.5 & & \\
\hline & sem & 2.1 & & \\
\hline & Min & 19.0 & & \\
\hline & Max & 75.0 & & \\
\hline \multicolumn{5}{|l|}{ Social habits } \\
\hline \multirow{2}{*}{ Current smoker } & Yes & 11 & 18.0 & \\
\hline & No & 50 & 82.0 & $<0.0001$ \\
\hline \multirow{4}{*}{ Cigarettes (\#/day) } & $11-20$ & 4 & 6.6 & \\
\hline & $21-30$ & 4 & 6.6 & \\
\hline & $31-40$ & 3 & 4.9 & \\
\hline & None & 50 & 81.9 & $<0.0001$ \\
\hline \multirow{2}{*}{ Duration of smoking (years) } & $\geq 5$ & 11 & 18.0 & \\
\hline & None & 50 & 82.0 & $<0.0001$ \\
\hline \multirow{3}{*}{ Former smoker } & Yes & 10 & 16.4 & \\
\hline & No & 44 & 72.1 & \\
\hline & Unknown & 7 & 11.5 & $<0.0001$ \\
\hline \multirow{2}{*}{ Duration former smoker (years) } & $\geq 2$ & 10 & 16.4 & \\
\hline & None & 51 & 83.6 & $<0.0001$ \\
\hline \multirow{2}{*}{ Duration since stopped smoking (years) } & $\geq 1$ & 10 & 16.4 & \\
\hline & None & 51 & 83.6 & $<0.0001$ \\
\hline \multirow{3}{*}{ Alcohol consumption (\#/week) } & $<1$ & 25 & 41.0 & \\
\hline & $>1$ & 28 & 45.9 & \\
\hline & None & 8 & 13.1 & 0.003 \\
\hline \multirow{3}{*}{ Diet } & Yes & 17 & 27.9 & \\
\hline & No & 43 & 70.5 & \\
\hline & Others & 1 & 1.6 & $<0.0001$ \\
\hline \multicolumn{5}{|l|}{ Oral hygiene } \\
\hline \multirow{2}{*}{ Oral hygiene (\#/day) } & $\leq 1 \mathrm{x} / \mathrm{d}$ & 10 & 16.4 & \\
\hline & $\geq 2 \mathrm{x} / \mathrm{d}$ & 51 & 83.6 & $<0.0001$ \\
\hline \multirow{2}{*}{ Flossing } & Yes & 30 & 49.2 & 0.90 \\
\hline & No & 31 & 50.8 & \\
\hline \multirow{3}{*}{ Flossing use (\#/month) } & $\geq 1-10$ & 11 & 18.0 & \\
\hline & $>10$ & 18 & 29.5 & \\
\hline & None & 32 & 52.5 & 0.04 \\
\hline \multirow{2}{*}{ Mouth wash } & Yes & 19 & 31.2 & \\
\hline & No & 42 & 68.9 & 0.003 \\
\hline & $\geq 4-16$ & 8 & 13.1 & \\
\hline Mouth wash (\#/month) & $\geq 30$ & 11 & 18.0 & \\
\hline & None & 42 & 68.9 & $<0.0001$ \\
\hline Bracesuse & Yes & 2 & 3.3 & \\
\hline Draces use & No & 59 & 96.7 & $<0.0001$ \\
\hline Denture use & Yes & 7 & 11.5 & \\
\hline Denture use & No & 54 & 88.5 & $<0.0001$ \\
\hline Oral problems & Yes & 14 & 23.0 & \\
\hline Oral problems & No & 47 & 77.1 & $<0.0001$ \\
\hline Acute/chronic diseases & & & & \\
\hline Continued & & & & \\
\hline
\end{tabular}




\begin{tabular}{|l|l|l|l|l|}
\hline Parameter & categories & Number $(\mathbf{n}=\mathbf{6 1})$ & percent & $P$ value \\
\hline \multirow{2}{*}{ Acute disease } & Yes & 4 & 6.6 & \\
\hline & No & 57 & 93.4 & $<0.0001$ \\
\hline \multirow{2}{*}{ Chronic disease } & Yes & 10 & 16.4 & \\
\cline { 2 - 5 } & No & 51 & 83.6 & $<0.0001$ \\
\hline Diagnostic and therapeutic procedures & & & & \\
\hline \multirow{2}{*}{ Radiological examinations (last 6 months) } & Ever & 11 & 18.0 & \\
\hline & Never & 50 & 82.0 & $<0.0001$ \\
\hline \multirow{5}{*}{ Type of radiological examinations (last 6 months, multiple entries) } & X-ray & 11 & 15.7 & \\
\cline { 2 - 5 } & CT-scan & 6 & 8.6 & \\
\cline { 2 - 5 } & Nuclear medicine & 1 & 1.4 & \\
\cline { 2 - 5 } & PET/SPECT & 2 & 2.9 & \\
\cline { 2 - 5 } & None & 50 & 71.4 & $<0.0001$ \\
\hline Radiotherapy & No & 61 & 100 & n.a \\
\hline
\end{tabular}

Table 1. The table summarizes the characteristics of all 61 donors such as demographics (e.g. sex, age), social habits (e.g. alcohol, cigarette consumption), oral hygiene (e.g. flossing, mouth wash), acute/chronic diseases and previous radiological procedures (e.g. number of CT-scans). Shown are the numbers per group with descriptive statistics where appropriate (n, mean, minimum [min], maximum [max], standard deviation [stdev] and standard error of the mean $[\mathrm{sem}])$, the percentage per category and the corresponding $P$ values (chi-square test).
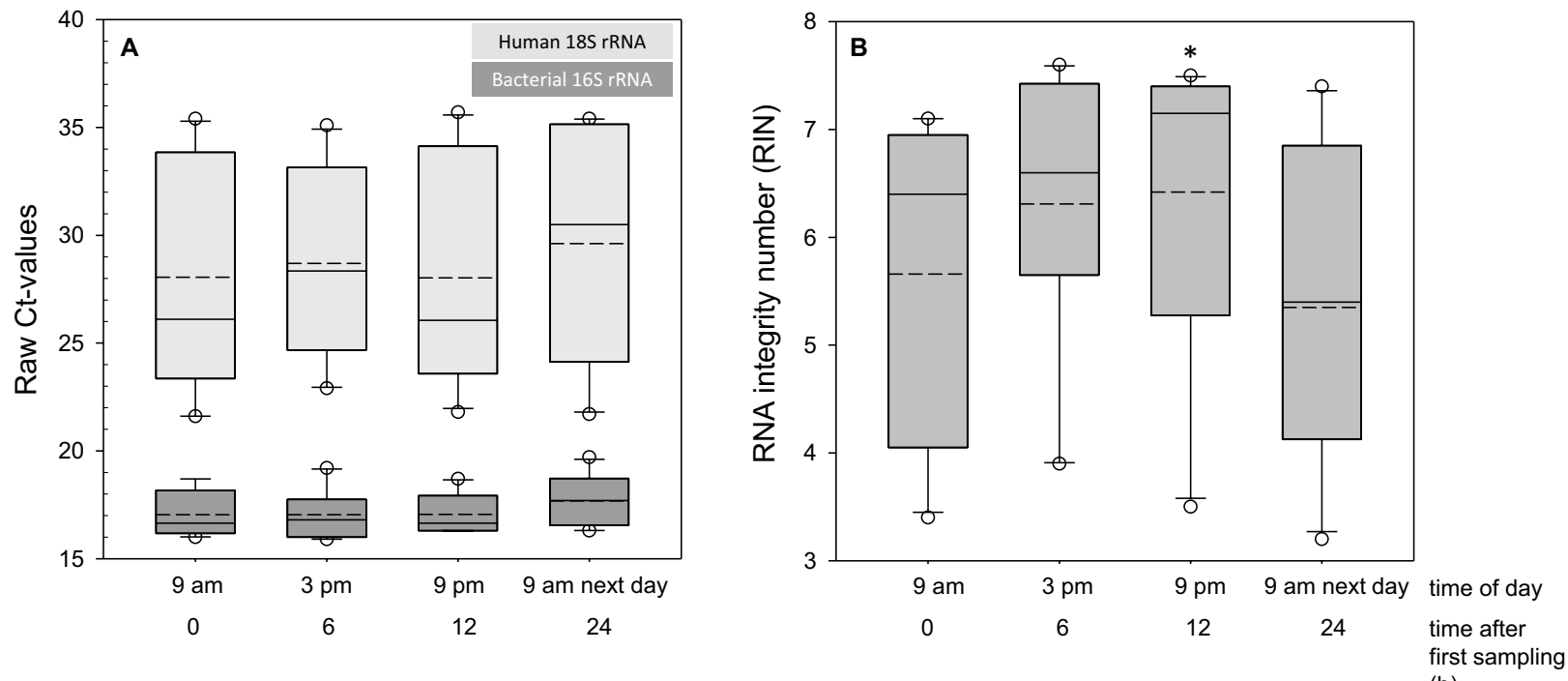

(h)

Figure 4. Box plots in (A) show the human $18 \mathrm{~S}$ rRNA and bacterial $16 \mathrm{~S}$ rRNA raw Ct-values for whole saliva samples (total $n=40$ ) per time point (each $n=10: 9 \mathrm{am}-0 \mathrm{~h} ; 3 \mathrm{pm}-6 \mathrm{~h} ; 9 \mathrm{pm}-12 \mathrm{~h} ; 9$ am next day-24h). The input amount for cDNA synthesis for each sample was $0.5 \mu \mathrm{g}$. The box plots in (B) represent the quality of isolated RNA using RNA integrity numbers $(\mathrm{RIN})$ for saliva samples (total $\mathrm{n}=40$ ) per time point (each $\mathrm{n}=10$ ). Dashed lines represent the mean, solid lines the median and dots the outliers. The asterisk ${ }^{*}$ ) refers to a $P$ value $<0.05$ using 9 am measurements as the reference.

indicating a pattern, caused by methodological reasons. A combination of ATP6, ACTB and B2M housekeeping genes (mean of Ct values) did not reduce inter-individual variability significantly (Fig. 6).

\section{Discussion}

The search for simple less-invasive sampling methods plays an important role for high-throughput diagnostic tests like for victims of radio/nuclear incidents. Besides whole blood, saliva, a non-invasive easily-accessible biofluid, has been shown to contain mRNA biomarkers for prediction and diagnosis of several diseases ${ }^{21,22}$. Examinations in this regard are challenged, because our previous studies indicate that most of the isolated RNA originates from the oral microbiome, thus, reducing the amount of isolated human RNA considerably. We previously modified the methodology to better analyse the low abundance of the human RNA fraction from whole saliva ${ }^{15}$. 


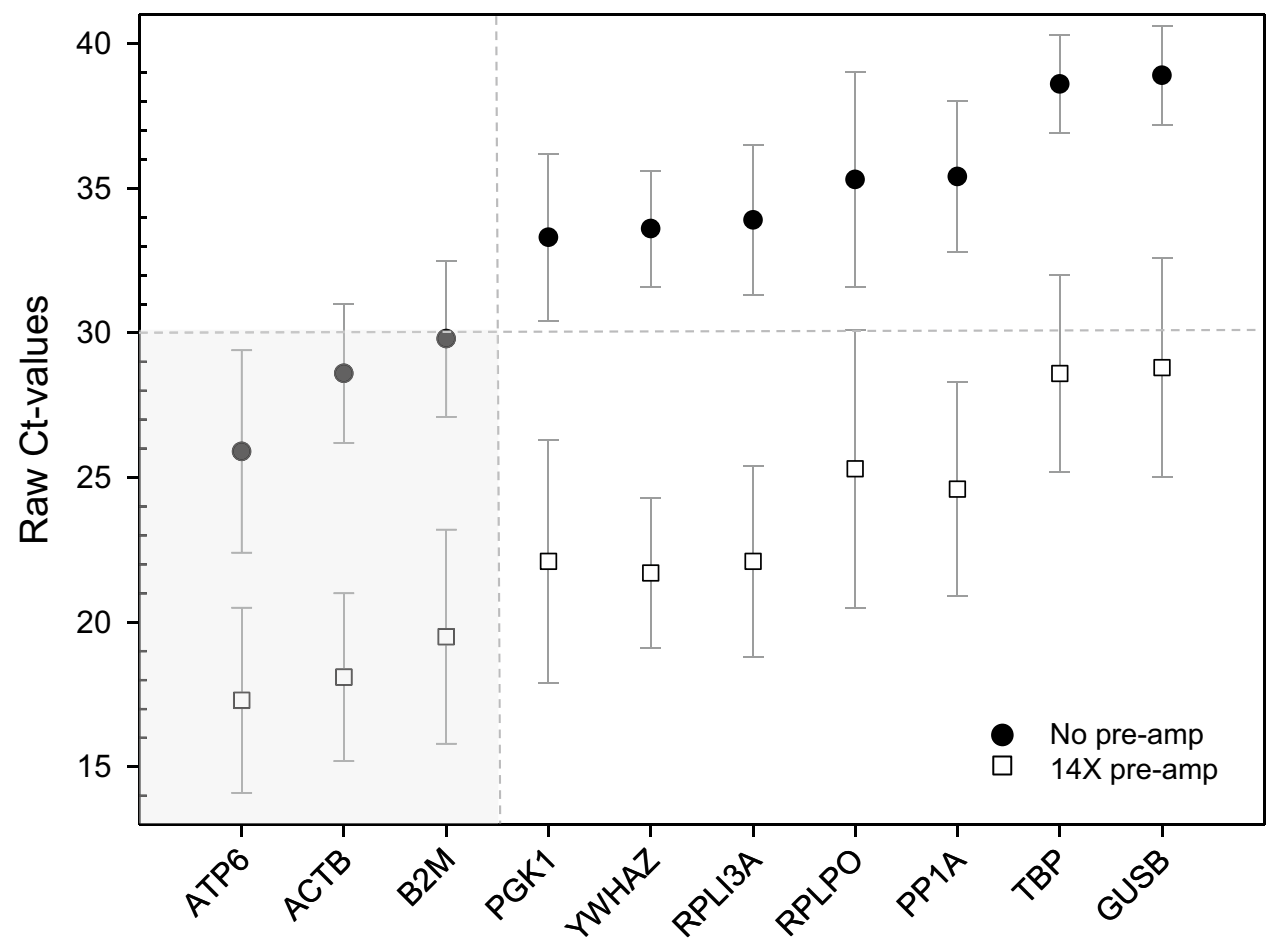

Figure 5. The graph represents raw Ct values (threshold cycles) of potential housekeeping genes $(n=10)$ analyzed for the 40 samples from 10 donors (four time points per donor), arranged in ascending order of raw unamplified Ct values. For each gene, the Ct values measured via qRT-PCR with cDNA without preamplification as well as after $14 \times$ pre-amplification is shown. Symbols represent geometric mean values and error bars reflect the standard error of mean per gene. Vertical and horizontal grey dashed lines show the cut-off (mean $\mathrm{Ct} \leq 30)$. Three of the genes $(A C T B, A T P 6$ and $B 2 M$, highlighted in grey area) showed un-amplified Ct-values $<30$ indicating that no pre-amplification for adequate detection will be required.

In the current study, we confirmed previous results increasing the sample size from 12 to 91 whole saliva samples. Furthermore, we modified the previously described workflow to ensure equal human RNA input for cDNA-synthesis as a prerequisite for comparability among samples when performing quantitative RT-PCR. In addition to normalization using a housekeeping gene, this normalization step via RNA quantification proved to be a robust method when measuring RNA expression between samples ${ }^{23}$. Spectrophotometrically, only total RNA (non-specific human and bacterial) can be measured. Measurement of total RNA quantity is relatively uninformative considering the high and inhomogeneous bacterial contamination of saliva samples. To resolve this issue, we relatively quantified human RNA using $18 \mathrm{~S}$ rRNA as surrogate and bacterial contamination using $16 \mathrm{~S}$ rRNA as surrogate, and introduced a correction factor for the same starting material (human RNA amount) for downstream cDNA-synthesis. By performing a second cDNA-synthesis with the High-capacity Kit (followed by qRT-PCR with detection of human $18 \mathrm{~S}$ rRNA and bacterial $16 \mathrm{~S}$ rRNA) we confirmed that we were able to adjust the amount of human RNA input for downstream cDNA synthesis with the SuperScript ${ }^{\oplus}$ III First-Strand Synthesis System (amount of starting material: $1 \mathrm{pg}-5 \mu \mathrm{g}^{19}$ ) to equal amounts.

In the current study we also examined factors with potential impact on RNA quantity and quality. Those included different collection time points for saliva sampling or extraneous factors, demographic and epidemiological. Concerning circadian periodic rhythmicity, published protocols from other research groups recommend highest yields of RNA as well as best quality when sampling between 9 and $11 \mathrm{am}^{9,24-28}$. Our study indicated no significant differences in RNA amounts between the collection time points, but intra-individual differences were high (> 1000-fold). Also, RIN values slightly improved at later ( $3 \mathrm{pm}$ and $9 \mathrm{pm}$ ) relative to early collection time points $(9 \mathrm{am})$, indicating that saliva samples can be collected during the whole day, thus, widening the applicability of this approach, e.g. for clinical use.

We did not observe any significant differences concerning sociodemographic and epidemiological characteristics that would explain the observed magnitude of inter-individual variance of human RNA yields. Furthermore, the differences in amounts of human RNA (raw Ct value for $18 \mathrm{~S}$ rRNA was $\leq 30$ ) between the samples could not be explained by sociodemographic or epidemiological characteristics. In this study, the addressed sociodemographic and epidemiological conditions seemed to be of minor relevance for interpretation of saliva gene expression results.

Human 18S rRNA, as a commonly known housekeeping gene, cannot be used as a normalizer in gene expression analysis in the current application due to the lack of a poly(A)+-tail ${ }^{15}$. We examined the baseline gene expression values of ten commonly used housekeeping genes. These genes appeared not or only weakly altered by sociodemographic or epidemiological factors which adds to their robustness. ATP6, ACTB and B2M appeared 

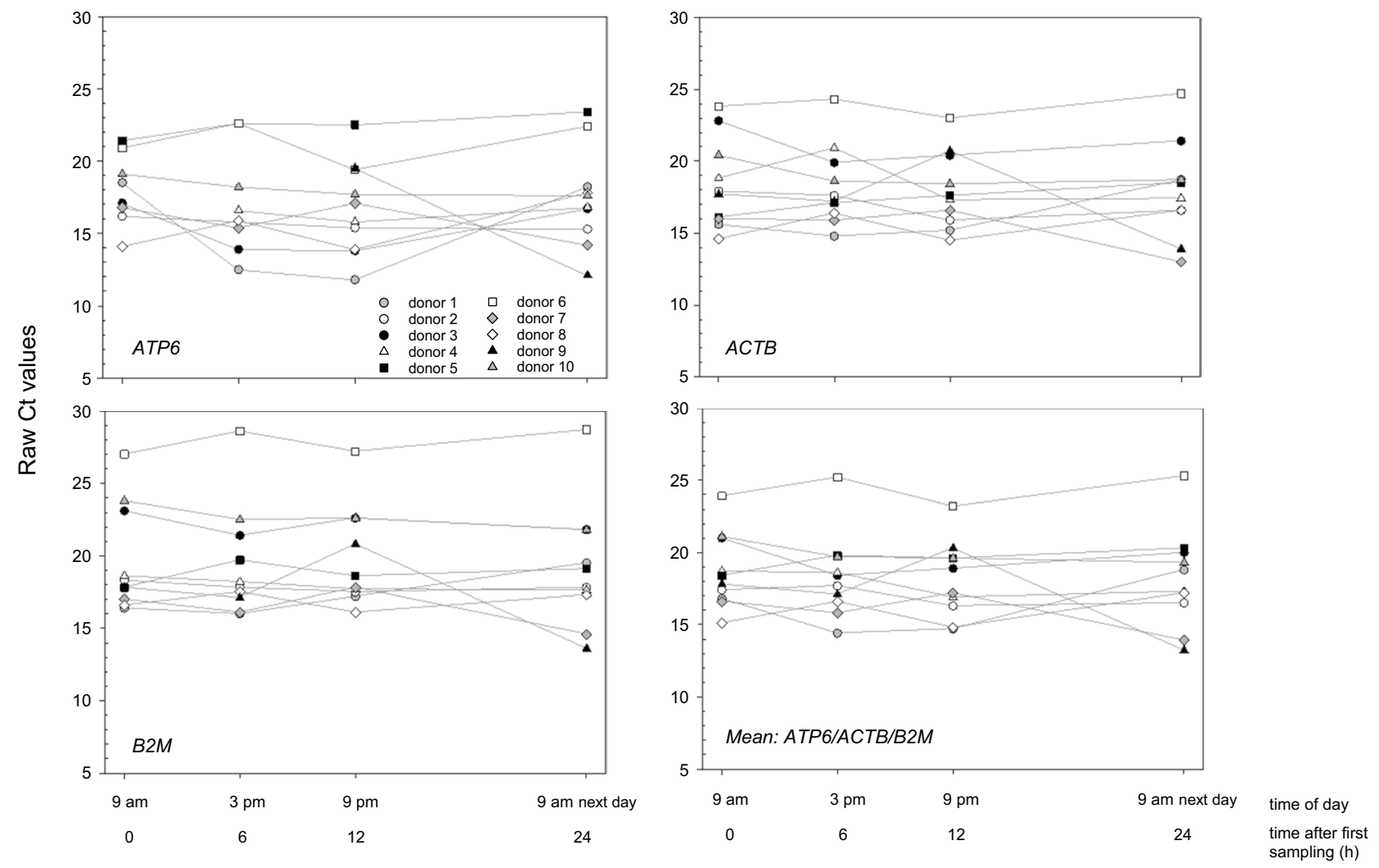

Figure 6. Raw Ct values (threshold cycles) of three candidate house-keeping genes (ACTB, ATP6 and B2M) as well as a combination of them (arithmetic mean) are depicted over time (four time point each: 9 am- 0 h; $3 \mathrm{pm}-6 \mathrm{~h} ; 9 \mathrm{pm}-12 \mathrm{~h} ; 9$ am next day $-24 \mathrm{~h}$ ) for each donor. They were fulfilling the criteria for being an appropriate housekeeping gene in this context.

most suitable, because sufficiently high copy numbers ensured detection in all samples indicating methodological robustness. However, inter-individual differences in gene expression and certain time points were found in several donors. These patterns occurred among all genes, suggesting they may be caused by methodological reasons. Combining all three housekeeping genes in our study did not reduce the variance significantly, but normalization based on more than one reference gene has been increasingly suggested by others ${ }^{29-31}$. Certainly, combining ATP6, ACTB and B2M as housekeeping genes for expression studies using human saliva will increase the robustness and, therefore, would be suggested. Nevertheless, planned future studies on saliva samples from irradiated donors will finally show whether radiation impacts these three identified genes, which would render them unsuitable as housekeeping genes. The applicability of suggested housekeeping genes has to be proven in future independent studies.

Finally, some limitations of this manuscript need to be considered. The major limitation lies in the fact that epidemiological data was gathered from the healthy donors by anamnesis. For example, the oral health status was self-reported and not confirmed by medical examination.

Nevertheless, a main strength of this study is that it represents a comprehensive examination of different facets when working with human whole saliva. The enhancement of the methodology and the proper examination of potential confounders like the influence of sociodemographic and epidemiologic characteristics that could potentially influence salivary isolates are completely novel findings, not described before in the literature. Considering saliva as an emerging source of body fluid for gene expression examinations underlines the importance of those findings. A key strength of the present study was the sample size: 91 samples from 61 donors in total are remarkable numbers considering molecular biological studies. These numbers and the numerous endpoints in this study are sufficient for creating reliable hypotheses.

In summary, we (I) improved the comparability of gene expression measurements among different saliva samples, (II) demonstrated that quality and quantity of RNA isolates is highly robust considering potential confounding factors such as demographics/epidemiologic and the saliva sampling time, making the approach of saliva collection even more attractive for further biomarker studies and (III) identified a set of potential housekeeping genes (ATP6, ACTB and B2M) and suggested their combination to increase robustness of saliva-based gene expression studies. 


\begin{tabular}{|c|c|c|c|c|c|c|c|}
\hline & \multicolumn{7}{|c|}{ raw Ct value } \\
\hline & $\mathbf{n}$ & Mean & stdev & sem & Min & Max & $P$ value \\
\hline \multicolumn{8}{|c|}{$P G K 1-$ no preamp } \\
\hline \multicolumn{8}{|c|}{ Alcohol consumption per week } \\
\hline None & 1 & 31.5 & & & 31.5 & 31.5 & \\
\hline$<1$ & 4 & 34.9 & 0.6 & 0.3 & 34.4 & 35.7 & \\
\hline$>1$ & 5 & 32.8 & 1.9 & 0.8 & 29.6 & 34.1 & 0.03 \\
\hline \multicolumn{8}{|c|}{$P G K 1-14 \times$ preamp } \\
\hline \multicolumn{8}{|c|}{ Mouth wash (\#/month) } \\
\hline None & 5 & 21.5 & 0.5 & 0.2 & 21 & 22.3 & \\
\hline$\geq 4-16$ & 2 & 16.6 & 3.3 & 2.3 & 14.3 & 19 & \\
\hline$\geq 30$ & 3 & 27.2 & 3.9 & 2.2 & 23.8 & 31.4 & 0.022 \\
\hline \multicolumn{8}{|c|}{$A C T B$-no preamp } \\
\hline \multicolumn{8}{|c|}{ Flossing } \\
\hline Yes & 7 & 29.3 & 2.2 & 0.8 & 27.5 & 33.6 & \\
\hline No & 3 & 26.8 & 0.4 & 0.2 & 26.4 & 27.2 & 0.02 \\
\hline \multicolumn{8}{|c|}{ Flossing (\#/month) } \\
\hline None & 3 & 26.8 & 0.4 & 0.2 & 26.4 & 27.2 & \\
\hline$\geq 1-10$ & 5 & 28.5 & 1.3 & 0.6 & 27.5 & 30.3 & \\
\hline$>10$ & 2 & 31.2 & 3.4 & 2.4 & 28.8 & 33.6 & 0.04 \\
\hline \multicolumn{8}{|c|}{$A C T B-14 \times$ preamp } \\
\hline \multicolumn{8}{|c|}{ Flossing (\#/month) } \\
\hline None & 3 & 16 & 1.6 & 0.9 & 14.6 & 17.7 & \\
\hline$\geq 1-10$ & 5 & 17.8 & 1.9 & 0.8 & 16 & 20.4 & \\
\hline$>10$ & 2 & 23.3 & 0.7 & 0.5 & 22.8 & 23.8 & 0.047 \\
\hline \multicolumn{8}{|c|}{ Mouth wash (\#/month) } \\
\hline None & 5 & 17.2 & 1.4 & 0.6 & 15.6 & 18.8 & \\
\hline$\geq 4-16$ & 2 & 15.4 & 1.1 & 0.8 & 14.6 & 16.1 & \\
\hline$\geq 30$ & 3 & 22.3 & 1.7 & 1 & 20.4 & 23.8 & 0.041 \\
\hline \multicolumn{8}{|c|}{$B 2 M-$ no preamp } \\
\hline \multicolumn{8}{|c|}{ Mouth wash } \\
\hline Yes & 5 & 31 & 1.7 & 0.8 & 29.3 & 33.7 & \\
\hline No & 5 & 28.2 & 0.8 & 0.4 & 27.1 & 29.4 & 0.016 \\
\hline \multicolumn{8}{|c|}{ Mouth wash (\#/month) } \\
\hline None & 5 & 28.2 & 0.8 & 0.4 & 27.1 & 29.4 & \\
\hline$\geq 4-16$ & 2 & 30.3 & 1.4 & 1 & 29.3 & 31.3 & \\
\hline$\geq 30$ & 3 & 31.4 & 2 & 1.2 & 29.9 & 33.7 & 0.0497 \\
\hline \multicolumn{8}{|c|}{$B 2 M-14 \times$ preamp } \\
\hline \multicolumn{8}{|c|}{ Flossing } \\
\hline Yes & 5 & 31 & 1.7 & 0.8 & 29.3 & 33.7 & \\
\hline No & 5 & 28.2 & 0.8 & 0.4 & 27.1 & 29.4 & 0.02 \\
\hline \multicolumn{8}{|c|}{ Flossing (\#/month) } \\
\hline None & 3 & 16.9 & 0.7 & 0.4 & 16.4 & 17.8 & \\
\hline$\geq 1-10$ & 5 & 19.1 & 2.7 & 1.2 & 17 & 23.8 & \\
\hline$>10$ & 2 & 25.1 & 2.8 & 2 & 23.1 & 27 & 0.048 \\
\hline$R P L P O-\mathrm{no}$ & ream & & & & & & \\
\hline Mouth wash & & & & & & & \\
\hline Yes & 4 & 33.3 & 2.3 & 1.2 & 30.7 & 36.3 & \\
\hline No & 3 & 37.8 & 1.4 & 0.8 & 36.4 & 39.1 & 0.034 \\
\hline Age (years) & & & & & & & \\
\hline$\geq 19-30$ & 3 & 37.8 & 1.4 & 0.8 & 36.4 & 39.1 & \\
\hline$>30-50$ & 4 & 33.3 & 2.3 & 1.2 & 30.7 & 36.3 & \\
\hline$>50$ & 0 & & & & & & 0.034 \\
\hline GUSB-14x & eam & & & & & & \\
\hline Mouth wash & & & & & & & \\
\hline Yes & 3 & 31.5 & 3.1 & 1.8 & 28.1 & 34.3 & \\
\hline No & 5 & 26.9 & 1 & 0.4 & 25.2 & 27.9 & 0.025 \\
\hline
\end{tabular}




\begin{tabular}{|c|c|c|c|c|c|c|c|}
\hline & \multicolumn{7}{|c|}{ raw Ct value } \\
\hline & $\mathbf{n}$ & Mean & stdev & sem & Min & Max & $P$ value \\
\hline \multicolumn{8}{|c|}{$P P 1 A-14 \times$ preamp } \\
\hline \multicolumn{8}{|c|}{ Mouth wash } \\
\hline Yes & 5 & 28.4 & 4 & 1.8 & 25.3 & 34.7 & \\
\hline No & 5 & 23.3 & 1.9 & 0.9 & 20.8 & 25.8 & 0.016 \\
\hline \multicolumn{8}{|c|}{ Mouth wash (\#/month) } \\
\hline None & 5 & 23.3 & 1.9 & 0.9 & 20.8 & 25.8 & \\
\hline$\geq 4-16$ & 2 & 25.6 & 0.5 & 0.4 & 25.3 & 26 & \\
\hline$\geq 30$ & 3 & 30.2 & 4.3 & 2.5 & 26.1 & 34.7 & 0.031 \\
\hline \multicolumn{8}{|c|}{ YWHAZ-14× preamp } \\
\hline \multicolumn{8}{|c|}{ Mouth wash } \\
\hline Yes & 5 & 23.8 & 3.1 & 1.4 & 20.1 & 27.4 & \\
\hline No & 5 & 20 & 0.9 & 0.4 & 19 & 21.2 & 0.047 \\
\hline \multicolumn{8}{|c|}{ Mouth wash (\#/month) } \\
\hline None & 5 & 20 & 0.9 & 0.4 & 19 & 21.2 & \\
\hline$\geq 4-16$ & 2 & 20.5 & 0.6 & 0.4 & 20.1 & 21 & \\
\hline$\geq 30$ & 3 & 26 & 1.3 & 0.8 & 24.8 & 27.4 & 0.049 \\
\hline \multicolumn{8}{|c|}{ ATP6-no preamp } \\
\hline \multicolumn{8}{|c|}{ Oral problems } \\
\hline Yes & 2 & 30.3 & 4.8 & 3.4 & 26.9 & 33.6 & \\
\hline No & 8 & 28.1 & 1.3 & 0.5 & 26.4 & 30.3 & 0.037 \\
\hline \multicolumn{8}{|c|}{ ATP6-14× preamp } \\
\hline \multicolumn{8}{|c|}{ Radiological examination (last 6 months) } \\
\hline Never & 6 & 18.9 & 1.9 & 0.8 & 16.8 & 21.4 & \\
\hline Ever & 2 & 15.1 & 1.5 & 1.1 & 14.1 & 16.2 & 0.046 \\
\hline \multicolumn{8}{|c|}{$R P L I 3 A-14 \times$ preamp } \\
\hline \multicolumn{8}{|c|}{ Mouth wash (\#/month) } \\
\hline None & 5 & 23.1 & 1.8 & 0.8 & 20.9 & 25.5 & \\
\hline$\geq 4-16$ & 2 & 19.2 & 1.3 & 0.9 & 18.3 & 20.1 & \\
\hline$\geq 30$ & 3 & 26.8 & 4 & 2.3 & 23.1 & 31.1 & 0.047 \\
\hline
\end{tabular}

Table 2. Overview of the housekeeping gene expression results (raw $\mathrm{Ct}$ values either without pre-amplification or after $14 \times$ pre-amplification) and the significant correlations with sociodemographic and epidemiological characteristics. Provided are numbers (n) per category and corresponding descriptive statistics: mean, minimum [min], maximum [max], standard deviation [stdev] and standard error of the mean [sem]. Comparisons of categorical variables with gene expression values were performed using the non-parametrical Kruskal-Wallis (KW) test.

Received: 7 April 2021; Accepted: 3 December 2021

Published online: 10 February 2022

\section{References}

1. Quinn, J. F. et al. Extracellular RNAs: Development as biomarkers of human disease. J. Extracell. Vesicles 4, 27495 (2015).

2. Li, M. H., Fu, S. B. \& Xiao, H. S. Genome-wide analysis of microRNA and mRNA expression signatures in cancer. Acta Pharmacol. Sin. 36, 1200-1211 (2015).

3. Condrat, C. E. et al. miRNAs as biomarkers in disease: Latest findings regarding their role in diagnosis and prognosis. Cells 9 , 276 (2020).

4. Szabo, D. T. Transcriptomic biomarkers in safety and risk assessment of chemicals. In Biomarkers in Toxicology (Ed. Gupta, R. C.) 1033-1038. https://doi.org/10.1016/B978-0-12-404630-6.00062-2 (Elsevier Inc, 2014).

5. Maron, J. L. et al. Neonatal salivary analysis reveals global developmental gene expression changes in the premature infant. Clin. Chem. 56, 409-416 (2010).

6. Watanabe, K., Akutsu, T., Takamura, A. \& Sakurada, K. Practical evaluation of an RNA-based saliva identification method. Sci. Justice 57, 404-408 (2017).

7. Lacombe, J. et al. Analysis of saliva gene expression during head and neck cancer radiotherapy: A pilot study. Radiat. Res. 188, 75-81 (2017).

8. Ghizoni, J. S., Nichele, R., de Oliveira, M. T., Pamato, S. \& Pereira, J. R. The utilization of saliva as an early diagnostic tool for oral cancer: microRNA as a biomarker. Clin. Transl. Oncol. 22, 804-812 (2020).

9. Li, Y. et al. Salivary transcriptome diagnostics for oral cancer detection. Clin. Cancer Res. 10, 8442-8450 (2004).

10. Kaczor-Urbanowicz, K. E. et al. Saliva diagnostics-Current views and directions. Exp. Biol. Med. 242, 459-472 (2017).

11. Chen, W., Cao, H., Lin, J., Olsen, N. \& Zheng, S. G. Biomarkers for primary Sjögren's syndrome. Genomics Proteomics Bioinform. 13, 219-223 (2015).

12. Michael, A. et al. Exosomes from human saliva as a source of microRNA biomarkers. Oral Dis. 16, 34-38 (2010).

13. Yoshizawa, J. M. et al. Salivary biomarkers: Toward future clinical and diagnostic utilities. Clin. Microbiol. Rev. 26, 781-791 (2013). 
14. Segal, A. \& Wong, D. T. Salivary diagnostics: Enhancing disease detection and making medicine better. Eur. J. Dent. Educ. 12, $22-29$ (2008).

15. Ostheim, P. et al. Overcoming challenges in human saliva gene expression measurements. Sci. Rep. 10, 11147 (2020).

16. Only, U. Oragene ${ }^{\bullet} \bullet$ RNA purification protocol using the Qiagen RNeasy Micro Kit for volumes up to $1000 \mu \mathrm{L}$. 3-5 (2012).

17. Life Technologies. mirVana ${ }^{\mathrm{TM}}$ miRNA Isolation Kit. 33 (2011).

18. Applied Biosystems. High Capacity cDNA Reverse Transcription Kits for 200 and 1000 Reactions Protocol (Rev E). Manual 1-29 (2010).

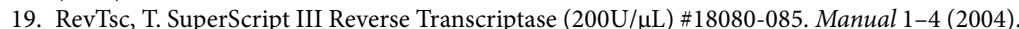

20. Fisher, T. \& July, S. TaqMan PreAmp Master Mix User Guide (2018).

21. Aro, K., Wei, F., Wong, D. T. \& Tu, M. Saliva liquid biopsy for point-of-care applications. Front. Public Health 5, 77 (2017).

22. Pernot, E., Cardis, E. \& Badie, C. Usefulness of saliva samples for biomarker studies in radiation research. Cancer Epidemiol. Biomark. Prev. 23, 2673-2680 (2014).

23. Dheda, K. et al. Validation of housekeeping genes for normalizing RNA expression in real-time PCR. Biotechniques 37, 112-119 (2004).

24. Palanisamy, V. \& Wong, D. T. Transcriptomic analyses of saliva. Methods Mol. Biol. 666, 43-51 (2010).

25. Navazesh, M. Methods for collecting saliva. Ann. N. Y. Acad. Sci. 694, 72-77 (1993).

26. Patel, R. S. et al. High resolution of microRNA signatures in human whole saliva. Arch. Oral Biol. 56, 1506-1513 (2011).

27. Park, N. J. et al. Salivary microRNA: Discovery, characterization, and clinical utility for oral cancer detection. Clin. Cancer Res. 15, 5473-5477 (2009).

28. Majem, B., Li, F., Sun, J. \& Wong, D. T. W. RNA sequencing analysis of salivary extracellular RNA. Methods Mol. Biol. 1537, 17-36 (2017).

29. Tricarico, C. et al. Quantitative real-time reverse transcription polymerase chain reaction: Normalization to rRNA or single housekeeping genes is inappropriate for human tissue biopsies. Anal. Biochem. 309, 293-300 (2002).

30. Vandesompele, J. et al. Accurate normalization of real-time quantitative RT-PCR data by geometric averaging of multiple internal control genes. Genome Biol. 3, 1-12 (2002).

31. Schmid, H. et al. Validation of endogenous controls for gene expression analysis in microdissected human renal biopsies. Kidney Int. 64, 356-360 (2003).

\section{Acknowledgements}

This study includes work from Selamawit Worku Alemu Master thesis. We highly appreciate the work of Thomas Müller, Sven Doucha-Senf and Oliver Wittmann, who carefully and patiently introduced SW Alemu in techniques performed in the course of this project and constantly supported her qRT-PCR experiments.

\section{Author contributions}

P.O. and M.A. wrote the main manuscript text and prepared the figures, tables and supplement table. A.T., S.W.A., T.P., G.W., S.G., S.A., M.H., C.S., S.S. and M.P. revised the work. A.T., I.S., M.D., M.M.S., G.K. contributed to the conception of the study. All authors reviewed the manuscript.

\section{Funding}

Open Access funding enabled and organized by Projekt DEAL. This work was supported by the German Ministry of Defence and the Czech Ministry of Defence (A long-term developmental plan 1011).

\section{Competing interests}

The authors declare no competing interests.

\section{Additional information}

Supplementary Information The online version contains supplementary material available at https:/doi.org/ 10.1038/s41598-022-05670-5.

Correspondence and requests for materials should be addressed to P.O.

Reprints and permissions information is available at www.nature.com/reprints.

Publisher's note Springer Nature remains neutral with regard to jurisdictional claims in published maps and institutional affiliations.

(c) (i) Open Access This article is licensed under a Creative Commons Attribution 4.0 International cc) License, which permits use, sharing, adaptation, distribution and reproduction in any medium or format, as long as you give appropriate credit to the original author(s) and the source, provide a link to the Creative Commons licence, and indicate if changes were made. The images or other third party material in this article are included in the article's Creative Commons licence, unless indicated otherwise in a credit line to the material. If material is not included in the article's Creative Commons licence and your intended use is not permitted by statutory regulation or exceeds the permitted use, you will need to obtain permission directly from the copyright holder. To view a copy of this licence, visit http://creativecommons.org/licenses/by/4.0/.

(c) The Author(s) 2022 